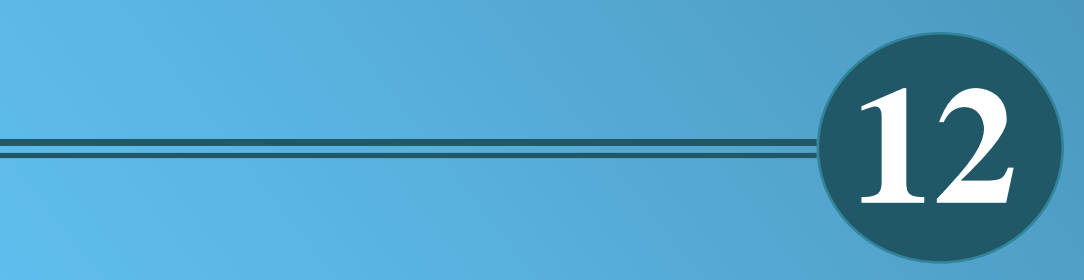

\title{
EFECTO DE DIFERENTES CONCENTRACIONES DE CREOSOTA LÍQUIDA EN LA GERMINACIÓN DE GENOTIPOS DE TOMATE (Lycopersicon esculentum Mill.)
}

Clotilde Andrade, Lady Rodríguez, Antonio Mora. 


\title{
Efecto de diferentes concentraciones de creosota líquida en la germinación de genotipos de tomate (Lycopersicon esculentum Mill.)
}

\section{Effect of different concentrations of liquid creosote on the germination of genotypes of tomato (Lycopersicon esculentum Mill.)}

\author{
Clotilde Andrade ${ }^{1}$, Lady Rodríguez ${ }^{2}$, Antonio Mora ${ }^{1}$ \\ 1 Universidad Estatal Península de Santa Elena (UPSE) Facultad de Ciencias Agrarias \\ 2 Unidad Educativa del Milenio "Cerezal Bellavista" \\ candrade@upse.edu.ec
}

\begin{abstract}
Resumen
En la provincia de Santa Elena, Ecuador, la producción anual de tomate riñón representa el 26,3\% del total nacional y, de acuerdo al INEC, en el año 2012 toda la cosecha se destinó a la venta; pero a partir de esa fecha, la superficie de siembra en las zonas productoras ha disminuido debido, principalmente, a factores abióticos como la sequía y la salinidad, que provocan alta incidencia de insectos-plaga y enfermedades. Bajo esta premisa, el presente estudio evaluó la germinación de tres genotipos de tomate bajo el efecto de cinco concentraciones de creosota líquida, en condiciones controladas de laboratorio, con el fin de obtener genotipos con características de tolerancia a las principales plagas que afectan al cultivo. Se consideró el porcentaje de germinación desde el día tres al 20, de acuerdo a la emergencia de cada uno en las diferentes concentraciones. El experimento fue llevado en un DCA en arreglo factorial 5x3, utilizando 100 semillas certificadas por tratamiento. Los resultados del ANDEVA revelaron diferencias estadísticas significativas en germinación al $1 \%$ de probabilidades para la interacción Genotipos por Concentraciones, destacándose G3 por germinar un 5\% en la concentración más alta al día 20 de la evaluación.
\end{abstract}

Palabras Claves: genotipos, concentraciones, creosota, germinación, tomate.

\begin{abstract}
In the province of Santa Elena, Ecuador, the annual production of kidney tomato represents $26.3 \%$ of the national total and, according to INEC, in 2012 all the harvest was destined for sale; but from that date on, the planting area in the producing areas has decreased, mainly due to abiotic factors such as drought and salinity, which cause a high incidence of insect-pests and diseases. Under this premise, the present study evaluated the germination of three tomato genotypes under the effect of five concentrations of liquid creosote, under controlled laboratory conditions, in order to obtain genotypes with tolerance characteristics to the main pests that affect the crop. The percentage of germination was considered from day three to 20, according to the emergence of each one in the different concentrations. The experiment was carried out in a DCA in factorial arrangement 5x3, using 100 seeds certified by treatment. The results of the ANDEVA revealed statistically significant differences in germination at $1 \%$ of the probabilities for the interaction Genotypes by concentrations, standing out G3 for germinating 5\% in the highest concentration at day 20 of the evaluation.
\end{abstract}

Kaywords: genotypes, concentrations, creosote, germination, tomato. 


\section{INTRODUCCIÓN}

En Santa Elena, la producción anual de tomate riñón representa el $26,3 \%$ a nivel nacional con una producción de 16694 toneladas, en una superficie sembrada de 572 ha, del cual, casi todo se lo destina a la venta, según el Instituto Nacional de Estadística y Censos, INEC (2012). En los últimos años, este cultivo ha disminuido en esta provincia debido, principalmente, a los fenómenos adversos como la sequía, la salinidad, excesiva radiación solar, que conducen a un clima desértico seco en la zona sur y en la parte norte del trópico sub-húmedo, lo que ha incidido en la presencia de insectos plagas y enfermedades causadas por hongos y bacterias, en el incremento de los costos de producción, ocasionado automáticamente la disminución de la superficie de siembra y, por ende, la producción de este cultivo (Ortiz, 2008).

De acuerdo al INEC (2013), para producir tomate en esta provincia, desde hace décadas se viene realizando el control químico convencional, dejando a un lado el manejo integrado de plagas y enfermedades, ocasionando la contaminación de los recursos naturales, eliminando los insectos benéficos y afectando el equilibrio ecológico, a más de los bajos rendimientos en la producción, con cifras de 1399 toneladas en una superficie sembrada de 86 ha, con perspectivas inciertas para los años venideros.

Tomando en cuenta lo antes mencionado, se consideró necesario probar en el presente estudio la germinación de genotipos de tomate, sometidas a diferentes concentraciones de creosota líquida, desinfectante de uso doméstico, partiendo del hecho, de haber realizado un scrining, en donde se utilizó 100 semillas recicladas de tomate, mismas que fueron sometidas, por 30 días, a la concentración pura de este desinfectante en cajas Petri; el resultado fue: ninguna semilla germinada. Sin embargo, se tomó al azar una de ellas, a continuación se le levantó la testa y se la observó en estereoscopio binocular, comprobándose que la radícula se había desarrollado y se encontraba intacta.

En la presente investigación se pretende seleccionar los genotipos de tomate que germinen en las concentraciones más altas de creosota líquida y obtener materiales con características de tolerancia a las principales plagas y enfermedades que afectan al cultivo en las diferentes zonas de producción de esta provincia.

\section{MATERIALES Y MÉTODOS}

El experimento se realizó bajo condiciones controladas de laboratorio en el laboratorio del Centro de Investigaciones Agropecuarias (CIAP) de la Facultad de Ciencias Agrarias de la Universidad Estatal Península de Santa Elena (UPSE). Los genotipos de tomate estudiados en la investigación fueron los híbridos Micaela HA 1903 (G1), Acerado 3059 (G2) y Daniela HA 144 (G3); el reactivo utilizado para definir las concentraciones fue la creosota líquida para uso doméstico, que se expende en el mercado nacional con el nombre de creolina, desinfectante de origen natural que se extrae de la destilación seca de la madera; de los vapores que se desprenden se obtiene la resina vegetal o esencia de trementina. El residuo que queda de este proceso es la creosota, masa de color oscuro, de aspecto siruposo, compuesto principalmente por fenol (ácido fénico) y cresol (ácido cresílico) que es la materia activa para elaborar diferentes compuestos para la limpieza y desinfección.

El diseño experimental para evaluar el poder germinativo de los genotipos de tomate, fue un DCA en arreglo factorial $5 \times 3$, es decir, cinco concentraciones de creosota y tres genotipos de tomate. Cada tratamiento consistió en la siembra de 100 semillas, previamente lavadas con agua destilada para separar el fungicida, con el que es tratada la semilla certificada. A continuación, cada tratamiento fue colocado en bandejas plásticas donde se aplicó, las siguientes concentraciones: 0,0 $\mathrm{mL}$ creosota $+100 \mathrm{~mL}$ agua destilada; $2,5 \mathrm{~mL}$ creosota $+97,5 \mathrm{~mL}$ agua destilada; $5,0 \mathrm{~mL}$ creosota + $95 \mathrm{~mL}$ agua destilada; 7,5 mL creosota $+92,5 \mathrm{~mL}$ agua destilada y $10,0 \mathrm{~mL}$ creosota $+90 \mathrm{~mL}$ agua destilada

La fórmula mediante la cual, se definieron las disoluciones de creosota para determinar el porcentaje del volumen de líquidos fue la siguiente:

$$
\% \text { volumen }=\frac{\text { volumen de soluto }(\mathrm{mL})}{\text { volumen de disolución }(\mathrm{mL})} \times 100
$$

La variable motivo del experimento fue el Porcentaje de germinación (\%). Para la evaluación de la misma se tomaron en consideración los cambios fisiológicos de la semilla a partir del tercer día, hasta los 20 días en que se observó la presencia de radícula e hipocótilo. 


\section{RESULTADOS Y DISCUCIÓN}

\subsection{PORCENTAJE DE GERMINACIÓN}

En el Cuadro 1 se presentan los resultados del análisis estadístico del factor A x B en función de los días evaluados en la germinación, observándose para la $\mathrm{F}$ calculada, diferencias estadísticas significativas al $1 \%$ para los días evaluados.

Cuadro 1. Análisis de la varianza ANDEVA de la interacción $A * B$ en la variable de germinación a los 3, 6, 10, 15 y 20 días.

\begin{tabular}{llcll}
\hline Días evaluados & Grados de Libertad & F. Calculada & $\mathbf{5 \%}$ & $\mathbf{1 \%}$ \\
\cline { 3 - 5 } & & & $\mathbf{5 \%}$ & 4,00 \\
\hline día 3 & 8 & $171,17^{* * *}$ & 2,64 & 4,00 \\
día 6 & 8 & $24,57^{* *}$ & 2,64 & 4,00 \\
día 10 & 8 & $7,98^{* *}$ & 2,64 & 4,00 \\
día 15 & 8 & $80,02^{* *}$ & 2,64 & 4,00 \\
día 20 & 8 & $131,49^{* *}$ & 2,64 & \\
\hline
\end{tabular}

Fuente: autores

En la Figura 1 se presentan los resultados del porcentaje germinación, de cada tratamiento, donde se observa que G1C1 (control) presenta valores que fluctúan entre el 91 y $94 \%$ para los días 3 y 6 de la evaluación, y para los días 10, 15 y 20, los valores máximos fueron del 97 al $100 \%$, mientras que para G2C1 se nota una disminución de germinación con valores de 75, 81, 87, 90 y $94 \%$ para los respectivos días evaluados; en el caso de G3C1 se presentan porcentajes significativos con valores que fluctúan entre 92 a $100 \%$, en los mismos días evaluados.

En cambio, cuando los mismos genotipos estuvieron expuestos a $\mathrm{C} 2(2,5 \mathrm{~mL}$ de creosota líquida) la germinación inició a partir del día 6 con valores de 26, 25 y $29 \%$ para G1, G2 y G3, respectivamente, mientras que para los mismos genotipos, a los días 10,15 y 20 , se reflejan valores entre 52 y $97 \%$.

Cuando los genotipos estuvieron bajo el efecto de C3 (5 mL de creosota líquida) se pudo notar, que la germinación se inició al día 10, excepto en G2 que obtuvo porcentajes bajos con valores de 8,12 y 17 $\%$ para en los días 10, 15 y 20, seguido del G1 que llegó a obtener valores más bajos aún de 11, 18 y 23 $\%$ respectivamente, siendo $\mathrm{G} 3$ el que sobresalió, al germinar con el 10 y $5 \%$ en las concentraciones más altas $\mathrm{C} 4$ y $\mathrm{C} 5$
Los resultados obtenidos en esta variable son similares a los que obtuvo Bonifa Nacimba (2001) cuando aplicó diferentes concentraciones de $\mathrm{NaCl}$ $(0,2,4,6$ dS.m-1) con el genotipo de tomate Amalia 95, encontrando diferencias significativas en los cuatro tratamientos, con la mayor cantidad de semillas germinadas en el control (98\%), con lo que demostró que en las concentraciones más altas disminuye la germinación de la semilla. De de manera similar, en el presente estudio G3 (control) obtuvo el mayor porcentaje de germinación con el $100 \%$, pero a medida que se aumentaron las concentraciones la germinación disminuyó, como es el caso de las concentraciones C3, C4 y C5 que se refieren a 5, 7,5 y $10 \mathrm{~mL}$ de creosota líquida.

A pesar de que en el presente experimento no se utilizó el cloruro de sodio como sustrato, las incidencias de las concentraciones de creolina resultan similares a los mencionados por Goykovic y Saavedra (2007), en el sentido de que los efectos producidos por la salinidad consisten en diferentes comportamientos de las plantas de tomate, siendo unos más desfavorables que otros, como es la disminución del porcentaje de germinación de las semillas por las altas concentraciones de sales que se encuentran en el medio donde son sembradas, 
prologando también el tiempo en que realizan este proceso.

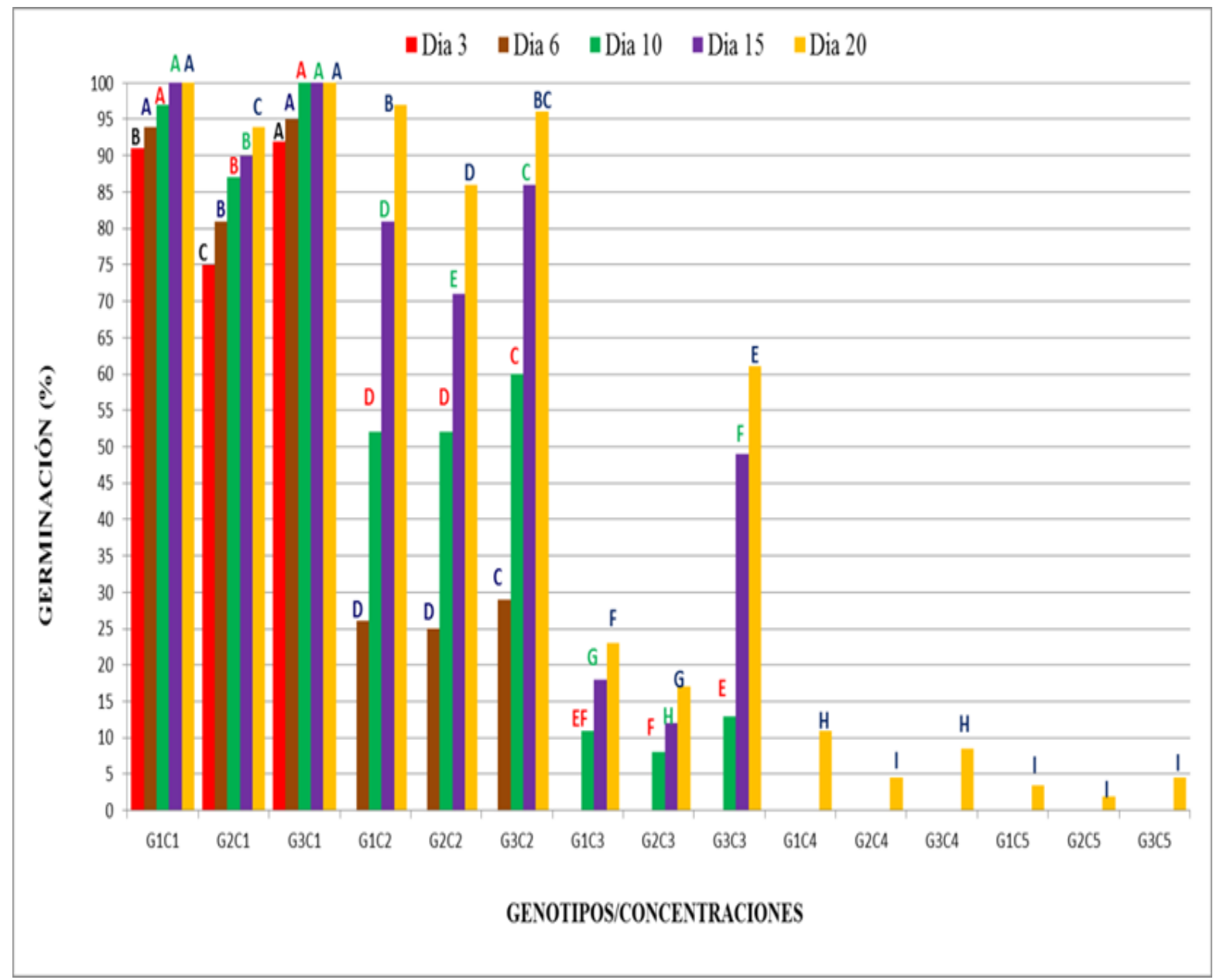

Figura 1. Porcentaje de germinación a los días 3, 6, 10, 15 y 20 de acuerdo a los genotipos/concentraciones

\section{CONCLUSIONES}

El genotipo G3 (Daniela HA 144), con un porcentaje del $5 \%$ de germinación, fue el mejor frente a las concentraciones más altas de creosota líquida, a los 20 días de la siembra de la semilla.

Los resultados indican que es posible tratar los semilleros de tomate con creosota líquida $\mathrm{y}$ mantener un porcentaje de germinación adecuado, lo que abre la posibilidad de que este desinfectante de origen natural y amigable con el ambiente, pueda ser utilizado con éxito en el control de plagas y enfermedades en el periodo de germinación de la semilla de tomate y de otras especies, lo que será motivo de nuevas investigaciones.

\section{REFERENCIAS BIBLIOGRÁFICAS}

Alvarado, P., 2009. Manual de cultivo de tomate (Lycopersicon esculentum Mill). Características botánicas. Nodo hortícola. Recuperado de: http://www.cepoc.uchile.cl/pdf/Manua_Cultivo_to mate.pdf.

Argerich, C.; Gaviola, JC., 2011. Manual de Producción de Semillas Hortícolas. Tomate. 
Desarrollo de la Semilla y la Germinación. 1ed. Argentina: INTA-EEA La Consulta.

Bemfica Steffen, R.; Antoniolli, Z.; Kist Steffen G.; Morales, D.; Pazzini Eckhardt, D.; Bassaco, A., 2010. Efeitos da creolina sobre a nematofauna associada a`cultura do fumo. Tecnológica. Revista del Departamento de Química y Física.

Benavides, P., 2015. Capacidad germinativa del genotipo de tomate (Lycopersicon esculentum Mill.) Floradade al estrés salino en diferentes fotoperiodos. Trabajo de Graduación de Ingeniero Agropecuario. Universidad Estatal Península de Santa Elena, ciudad La Libertad, Ecuador.

Blancard, D.; Laterrot, H.; Marchoux, G., 2011. Enfermedades del tomate: identificar, conocer, controlar. Editorial: Mundi-Prensa. Recuperado de Biblioteca Virtual Universidad Estatal Península Santa Elena.: http://www.ebrary.com.

Nacimba Bonifa, MP., 2011. Efecto de tres concentraciones de cloruro de sodio $(\mathrm{NaCl})$ en variables del crecimiento y desarrollo de semillas de tomate (Solanum lycopersium L.), variedad Amalia 95 en condiciones de laboratorio. Tesis de Diplomado. Universidad Técnica de Cotopaxi., Latacunga, Ecuador.

Camejo, D.; Torres, W., 2007. La salinidad y su efecto en los estadios iniciales del desarrollo de dos cultivares de tomate (Lycopersicon esculentum, Mill). Cultivos Tropicales. Vol. 21 Nro. 2. 2000. Cuba: Editorial Universitaria. Recuperado de Biblioteca Virtual Universidad Estatal Península Santa Elena: http://www.ebrary.com.

Corpeño B., 2004. Manual de Cultivo del Tomate Riñón. El Salvador.

Chamorro Amar CM., 2003. Efecto de la aplicación foliar de óxido de calcio sobre la clorosis marginal en hojas jóvenes de tomate. Fortaleza bajo invernadero frio. Recuperado de: http://ucv.altavoz.net/prontus_unidacad/site/.../cha morro_cristian.pdf

Días Pérez, T.; Alfonso Hernández, D., 2003. Comportamiento de la germinación de semillas de tomate tratadas con cloro $(\mathrm{Cl})$. Instituto de Investigaciones Hortícolas "Liliana Dimitrova". La Habana, Cuba.

C. Andrade/L. Rodríguez/A. Mora
Encuesta de Superficie y Producción Agropecuaria Continua ESPAC EC., 2013. Superficie, Producción y Ventas, según cultivos transitorios.

FAO, 2000. Producción mundial del tomate. Recuperado de: http://www.hortoinfo.es/index.php/noticias/1543tomate-mundo-15-07

FAO, 2012. Producción mundial del tomate. Recuperado de: http://www.hortoinfo.es/index.php/noticias/3084tomate-mundo-100314

Fundación Hondureña de Investigación Agrícola FHIA, 2011. Evaluación de estrategias para el control de la Hypsipyla grandella (Zeller) en la caoba. Hoja Técnica. № 10. Cortes, Honduras.

Giardina, EB.; Heredia, OS.; Castro, MA. \& Effron, DN., 2012. Fitotoxicidad del cromo sobre Phaseolus vulgaris L. Revista Agronomia y ambiente. Buenos Aires. Argentina.

Goykovic, V.; Saavedra, G., 2007. Algunos efectos de la salinidad en el cultivo del tomate y prácticas agronómicas de su manejo. Idesia. Chile. Vol. 25, $\mathrm{N}^{\mathrm{o}}$ 3. $47-58$.

Jaramillo, J.; Rodríguez, V.; Guzmán, M.; Zapata, M.; Rengifo, T., 2007. Manual técnico: buenas prácticas agrícolas en la producción de tomate bajo condiciones protegidas. CORPOICA. MANA. Gobernación de Antioquia-FAO.

Instituto Nacional de Investigaciones Agropecuaria INIAP, 2001. Respuesta de seis híbridos de tomate riñón (Lycopersicom esculentum), a dos distancias de siembra bajo invernadero, con manejo orgánico. El Altar - Chimborazo. Recuperado de: http://mail.iniapecuador.gov.ec/isis/view_detail.php $? \mathrm{mfn}=2317 \& q$ type $=$ query $\&$ dbinfo $=$ TESIST $\&$ word $s=$ HIBRIDOS

Instituto Nacional de Estadísticas y Censos INEC. Encuesta de Superficie y Producción Agropecuaria Continua. 2012. Recuperado de: http://www.ecuadorencifras.gob.ec/wpcontent/d escargas/Presentaciones/PRESENTACION-

Espac.pdf.

Instituto de Nutrición de Centro América y Panamá INCAP, 2006. Manual 8. Serie I. Ecotecnologías para la Producción Agrícola y Pecuaria. Recetas para el control de Insectos. 
Killian, SE.; Leiva, M., 2005. Efecto de sales de sodio y potasio sobre la germinación de semillas de tomate (Lycopersicum esculentum Mill.) y albahaca (Ocimum basilicum L.). Effect of sodium and potassium salts on tomato (Lycopersicum esculentum Mill.) and sweet basil (Ocimum basilicum L.) seed germination. Revista del Cizas, 6: 1-2.

Nuez, F., 2001. El cultivo del tomate. Ed. Grupo Mundi-Prensa.

Muñoz, M., 2001. Estudio de población, monitoreo y control del picudo negro (Cosmopolites sordidus, Germar) en el cultivo del plátano (Musa AAB). Recuperado de: http://bdigital.zamorano.edu/bitstream/11036/2382/ 1/T1342.pdf

Mendoza, M. y Proaño, J., 2008. Evaluación del efecto de tres niveles de N-P-K y dos de biofertilizantes a través del fertirriego en el cultivo de tomate (lycopersicum esculentumm.) en la zona de Daular, provincia del Guayas. Recuperado de: http://www.secsuelo.org/XICongreso/Simposios/N utricion/Presentacion/Ponencias/2.\%20Ing.\%20Ma ximo\%20Mendoza.pdf.

Monardes, H., 2009. Manual de cultivo de tomate (Lycopersicon esculentum Mill). Características botánicas. Nodo hortícola. Recuperado de: http://www.cepoc.uchile.cl/pdf/Manua_Cultivo_to mate.pdf

Montenegro, V., 2012. Evaluación de la aclimatación de veinte y dos cultivares de tomate (lycopersicumesculentummill), bajo invernadero, en Chugllin, cantón Chambo, provincia Chimborazo. Recuperado de: dspace.espoch.edu.ec/.../13T0757\%20MONTENE GRO\%20VICTOR.doc.

Osorio Díaz, D., 2003. Volvamos al campo. Producción de pimiento, tomate y lechuga en hidroponía. Universidad Nacional de Colombia. Ed. Grupo Latino Ltda.

Ortiz, F., 2008. Potencialidades Península Santa Elena. Ministerio de coordinación de desarrollo social. Subsecretaria de Inclusión Económica. Santa Elena. Ecuador.
Proquimsa, 2007. Hoja de Seguridad de materiales. Recuperado de: http://portalweb.ucatolica.edu.co/easyWeb2/files/5 6_12728_creolina.pdf

Rodríguez, D.; Montilla, J., 2002. Disminución de la marchitez causada por Fusarium en tomate con extracto de Citrus paradisi. Manejo Integrado de Plagas. Universidad Centroccidental "Lisandro Alvarado". Postgrado de Fitopatología. Apartado 400. Barquisimeto, Estado Lara, Venezuela. $\mathrm{N}^{\circ} 63$. $46 \mathrm{p}$.

Siavichay, M., 2011. Aclimatación de 10 cultivares de tomate (Lycopersicon esculentum Mill.), en el cantón Riobamba, provincia de Chimborazo. Escuela Superior Politécnica de Chimborazo. Facultad de Recursos Naturales. Riobamba, Ecuador. Recuperado de: file:///E:/Proyecto\%20tomate/Tesis\%20de $\% 2010 \%$ 20hib\%20tomate $\% 2013$ T0705\%20SAVICHAY\%2 0MAR\%C3\%8DA.pdf

Urbina, C., 2009. Manejo Integrado de las Principales Plagas y Enfermedades. Recuperado de: http://inta.gob.ar/documentos/manual-deproduccion-de-semillas-horticolas.tomate/at_multi_download/file/6.\%20cap.\%206desarrollo $\% 20 \mathrm{de} \% 201 \mathrm{a} \% 20$ semilla $\% 20 \mathrm{y} \% 201 \mathrm{a} \% 20$ germinaci\%C3\%B3n.pdf).

Villao, R., 2015. Capacidad germinativa de semillas de tomate (Lycopersicon esculentum Mill.) provenientes del agricultor al estrés osmótico en diferentes fotoperiodos. Trabajo de Graduación de Ingeniero Agropecuario. Universidad Estatal Península de Santa Elena, Ciudad La Libertad, Ecuador.

Wilson, C.; Loomis, W. Botánica. Trad IL de Coll. Limusa México. 2000. 682.

Zamorano, C.; Fuentes, CL., 2005. Potencial alelopático de Brassica campestris subsp. Rapa y Lolium temulentum sobre la germinación de semilla de tomate. Agronomía Colombiana. Vol. $23 \mathrm{~N}^{\circ} 2$. Bogotá. Recuperado de: http://eds.b.ebscohost.com. Biblioteca virtual Universidad Estatal Península Santa Elena. 